\title{
Constraining the gravitational coupling of axion dark matter at LIGO
}

\author{
Sunghoon Jung $\odot,{ }^{1, *}$ TaeHun Kim $\odot,{ }^{1, \uparrow}$ Jiro Soda, ${ }^{2, \$}$ and Yuko Urakawa $\odot^{3,4, \S}$ \\ ${ }^{1}$ Center for Theoretical Physics, Department of Physics and Astronomy, \\ Seoul National University, Seoul 08826, Korea \\ ${ }^{2}$ Department of Physics, Kobe University, Kobe 657-8501, Japan \\ ${ }^{3}$ Fakultüt für Physik, Universität Bielefeld, Bielefeld 33501, Germany \\ ${ }^{4}$ Department of Physics and Astrophysics, Nagoya University, Chikusa, Nagoya 464-8602, Japan
}

(Received 13 March 2020; accepted 21 August 2020; published 16 September 2020)

\begin{abstract}
The axion-gravity Chern-Simons coupling is well motivated but is relatively weakly constrained, partly due to difficult measurements of gravity. We study the sensitivity of LIGO measurements of chirping gravitational waves (GWs) on such coupling. When the frequency of the propagating GW matches with that of the coherent oscillation of axion dark matter field, the decay of axions into gravitons can be stimulated, resonantly enhancing the GW. Such a resonance peak can be detected at LIGO as a deviation from the chirping waveform. Since all observed GWs will undergo similar resonant enhancement from the Milky Way (MW) axion halo, LIGO O1 + O2 observations can potentially provide the strongest constraint on the coupling, at least for the axion mass $m_{a}=5 \times 10^{-13}-5 \times 10^{-12} \mathrm{eV}$. Along the course, we also emphasize the relevance of the finite coherence of axion fields and the ansatz separating forward and backward propagations of GWs. As a result, the parity violation of the Chern-Simons coupling is not observable from chirping GWs.
\end{abstract}

DOI: 10.1103/PhysRevD.102.055013

\section{INTRODUCTION}

The axion is an important candidate of dark matter. Axions are not restricted to the QCD axion, but a variety of axions are predicted from stringy setups [1]. They are very light pseudoscalar particles coupling to Chern-Simons terms of some gauge fields $F \tilde{F}$. Combined with proper cosmological histories, a wide range of axions can be a full dark matter candidate (see, e.g., [2,3]).

However, the axion is very elusive as it couples to standard model particles very weakly, suppressed by its large decay constant $f_{a}$. Thus, usual direct detection experiments are not sensitive to the axion. A whole new variety of axion detection experiments and astrophysical probes has been proposed, mainly based on its lightness (due to the pseudo Goldstone nature) and the coherent oscillation (due to the nonrelativistic dark matter nature) [4]. They can constrain the axion couplings to photons and electrons, for example through supernova cooling,

\footnotetext{
*sunghoonj@snu.ac.kr

†imthcha@snu.ac.kr

jiro@phys.sci.kobe-u.ac.jp

surakawa.yuko@h.mbox.nagoya-u.ac.jp
}

Published by the American Physical Society under the terms of the Creative Commons Attribution 4.0 International license. Further distribution of this work must maintain attribution to the author(s) and the published article's title, journal citation, and DOI. Funded by SCOAP ${ }^{3}$. oscillating electric dipole moments, birefringence of pulsars, quasars, and cosmic microwave background (CMB), and the mixing with the photon inside electron plasma. We refer to [5] for reviews.

But the axion-gravity coupling is relatively weakly constrained. Similarly to the axion-photon $a F \tilde{F}$ coupling, the axion-gravity Chern-Simons coupling can be generically produced $[6,7]$. Whenever there is a gravitational anomaly, there must exist an associated axion coupling to gravity. The latest bound on the axion-gravity coupling $\ell \lesssim 10^{8} \mathrm{~km}$ [Eq. (7)] comes from the measurement of frame-dragging effects around the Earth by Gravity Probe B [8].

In the meantime, the chirping gravitational wave (GW) from a binary merger arises as a new tool to probe the Universe. Since it has a well predicted waveform chirping in time and frequency domains in a particular way, even small perturbations to the chirping can be confidently detected. Example studies with dark matter perturbations are [9-14], one of which is probing coherently oscillating light dark matter around binary mergers [15].

In this paper, we study how the chirping GW can be perturbed by the coherent axion field as the GW propagates through it. Although the gravitational perturbation is usually very small, a resonant phenomenon can occur when the GW frequency matches with the axion Compton frequency. The resulting signal is a resonance peak in the frequency spectrum.

The resonant phenomenon on the electromagnetic (EM) wave has been studied with various observables. 
For example, the modification of the EM wave propagating through the coherent axion dark matter field can produce a sharp resonance peak in the frequency spectrum [16-20] or can even produce an echo coming back to us [21]. The resonance can also destabilize axion structures [22-24], possibly leaving some signals in the background or producing an explosive burst [25].

On the other hand, the GW resonance from the coherent dark matter field has not been studied in detail, even though the axion-gravity coupling is well motivated too. Up to our knowledge, the GW resonance was first studied in [26], but it lacks detailed analysis of realistic observables. Our work aims at providing an elaborate analysis for the GW resonance and its use for probing axion-gravity couplings with the LIGO. We will mainly focus on the modification of chirping GWs, but will discuss the instability of axion substructures too. Readers may also refer to [27] for resonance-induced GW bursts coming from dense axion structures, [28] for other non-resonant GW observables, and [29] for axion-generated GW background.

Our work also improves upon the previous works on the resonance in that we correctly include the finite spatial coherence of the axion field and separate the forward and backward waves. Although similar analyses have been done for EM waves in [17,21], the former ignored the spatial coherence while the latter did not discuss the forward wave. Both treatments are crucial in the LIGO observation, and one remarkable consequence is the absence of parity violation observables.

The paper is structured as follow. We start with a summary of the main points and physics of the paper in Sec. II. We derive and solve wave equations in Sec. III, introduce our axion signals on the chirping GW in Sec. IV, and present expected LIGO bounds and prospects in Sec. V. We provide further details on the resonance with various viewpoints in Sec. VI, and discuss interesting findings on the absence of parity violation in Sec. VII. Then we conclude in Sec. VIII.

\section{OVERVIEW}

We consider the MW axion halo, which is a highly coherent superposition of axion waves, with a long spatial coherence $\sim 1 / m_{a} \Delta v$ and a much longer temporal coherence (longer than the duration of the chirping $\mathrm{GW}$ in the LIGO band). The long coherence stems from the nonrelativistic nature $(v \sim \Delta v \ll 1)$ of the axion dark matter.

The coherent (temporal) oscillation can induce resonant enhancement of the chirping GW, when the GW frequency matches with the axion Compton frequency. Since the waveform of the chirping GW is very well predicted, the resonance peak can be detected. It can be further distinguished from accidental noise because all observed GWs will experience a similar phenomenon from the MW axion halo. We found that the correlation of all $11 \mathrm{GW}$ observations at LIGO $\mathrm{O} 1+\mathrm{O} 2$ can potentially provide one of the strongest constraints on the axion-gravity coupling.

The resonant phenomenon is essentially the stimulated decay of axions, although we treat those waves classically. We present several analyses to make sense of the particlelike interpretation of the solution of wave equations that we actually obtain and use.

The finite (spatial) coherence does impact the signal. Not only does it reduce the enhancement, but it also broadens the frequency width and induces finite time duration and time delay of the resonance peak. All these featuresresonance frequency, width, amplification, duration, and time delay-must be exploited in the search. Although we use only frequency-domain features in our estimation, we discuss possible discovery analysis utilizing the full features in the Appendix B.

We distinguish forward and backward-going GWs generated from the propagation through an axion halo. First, only forward waves coming toward us will be observed. Second, backward waves must be generated by the energymomentum conservation, if forward waves are to be enhanced. Last, mostly only forward and backward waves are generated, which can be understood from a symmetry consideration well inside a halo.

The distinction of forward and backward waves leads to different observable relations of the parity violation. In our case, the parity violation exists only on backward waves, hence not observable. But in existing studies, parity violation was observable because nonresonant regime was considered and/or stochastic waves were considered where the forward/backward distinction is not possible.

\section{PROPAGATION THROUGH COHERENT AXIONS}

We solve coupled wave equations between axion fields and GWs by using an ansatz suitable for the propagating GW. Then we discuss the solution near a resonance regime with small enhancement.

\section{A. Coupled wave equations}

The gravitational Chern-Simons Lagrangian $\mathcal{L}=\frac{\alpha}{4} a R \tilde{R}$ gives the linearized action in the flat background as (ignoring the cosmic expansion)

$$
\begin{aligned}
S_{\mathrm{EH}} & +S_{\mathrm{CS}} \\
= & \frac{\kappa}{4} \int d^{4} x\left[h^{i}{ }_{j, t} h^{j}{ }_{i, t}-h^{i}{ }_{j, k} h^{j}{ }_{i,}{ }^{k}\right. \\
& \left.-\frac{\alpha}{\kappa} \dot{a} \epsilon^{i j k}\left(h^{l}{ }_{i, t} h_{k l, j t}-h^{l}{ }_{i,}{ }^{m} h_{k l, m j}\right)\right],
\end{aligned}
$$

where $\alpha$ is the gravitational Chern-Simons coupling constant, $h_{i j}$ is the metric perturbation and $\kappa=1 / 16 \pi G$. 
Varying this action with respect to the metric perturbation $h_{i j}$ gives the wave equation [30,31]

$$
\left(\partial_{t}^{2}-\vec{\nabla}^{2}\right) h^{j}{ }_{i}=\frac{\alpha}{\kappa} \epsilon^{l k j}\left(\ddot{a} h_{k i, l t}+\dot{a} h_{k i, l t t}-\dot{a} h_{k i, m}{ }^{m}{ }_{l}\right) .
$$

We approximate the axion field $a$ to have only time dependence through its Compton oscillation (spatially homogeneous)

$$
a(t)=\frac{a_{0}}{2} e^{-i m_{a} t}+\text { c.c. }
$$

where $a_{0}$ is the complex amplitude, containing the initial phase information. As axions are nonrelativistic dark matter candidate, their small kinetic energy contribution to the Compton frequency is neglected. The amplitude $a_{0}$ (and hence the energy density) is assumed to be constant in time, as the energy density of the axion field is much larger than that of the chirping GW (Sec. VI A). For a more realistic axion halo spatial profile, see Sec. IV C.

To solve Eq. (2) for the propagating GW in a finite axion halo, we introduce an ansatz for $h_{i j}$ considering $^{1}$

(1) Plane waves propagating in the $\hat{z}$ direction.

(2) Backward wave. The conservation of momentum enforces the generation of backward propagating waves when the forward wave is enhanced. ${ }^{2} \mathrm{We}$ will distinguish forward and backward waves, in order to describe forward propagating GWs that we eventually observe. This leads to different observable relations from previous works; see Sec. VII.

(3) Circular polarization. The Levi-Civita tensor $\epsilon^{i j k}$ mixes the + and $\times$ polarizations, while right handed (R) and left handed (L) circular helicities are decoupled.

These conditions give the following ansatz, similar to the photon ansatz introduced in [17] but in the circular polarization basis:

$$
h_{i j}(z, t)=h_{i j}^{(R)}(z, t)+h_{i j}^{(L)}(z, t),
$$

where each helicity mode is expressed as ${ }^{3}$

\footnotetext{
${ }^{1}$ In Appendix A, we present another approach of solving the wave equation, giving the same result.

${ }^{2}$ From a symmetry consideration, the generation of only forward and backward waves must be true, at least well inside a finite halo. But there can be slight leakage over all directions near the boundary of a halo or a coherent patch, although the boundary still varies smoothly over a large scale. We ignore the leakage.

${ }^{3}$ The ansatz with $e^{-i \frac{m a}{2} t}$ rather than $e^{-i \omega t}$ is more convenient to solve the equation, but we will check this ansatz gives the correct dispersion relation; see below Eq. (16).
}

$$
\begin{aligned}
h_{i j}^{(s)}(z, t)= & \hat{e}_{i j}^{(s)} h_{F}^{(s)}(t) e^{i\left(k z-\frac{m_{a}}{2} t\right)} \\
& -i \hat{e}_{i j}^{(\bar{s})} h_{B}^{(s)}(t) e^{i\left(-k z-\frac{m_{a}}{2} t\right)}+\text { c.c. },
\end{aligned}
$$

where $h_{F}$ and $h_{B}$ are complex amplitudes for the forward and the backward waves, with the superscript $s=L, R$ denotes helicity and $\bar{s}$ refers to the opposite to $s$. The polarization tensor $\hat{e}_{i j}^{(s)}$ is defined with respect to the direction of $+\hat{z}$ propagation.

Applying Eqs. (3)-(5) into the wave equation (2) (with $\left.\left|\ddot{h}_{F, B} / \dot{h}_{F, B}\right| \ll m_{a}\right)$ gives coupled first-order differential equations for forward and backward waves. They become decoupled in the second order equations as

$$
\begin{aligned}
\ddot{h}_{F / B}^{(s)}(t) & =\left(\frac{m_{a}}{2}\right)^{2}\left(2 \pi G \ell^{4} m_{a}^{4} \frac{\left|a_{0}\right|^{2}}{4}-\epsilon^{2}\right) h_{F / B}^{(s)}(t) \\
& =\left(\frac{m_{a}}{2}\right)^{2}\left(\gamma^{2}-\epsilon^{2}\right) h_{F / B}^{(s)}(t) \\
& =\mu^{2}(\epsilon) h_{F / B}^{(s)}(t),
\end{aligned}
$$

where

$$
\ell^{2} \equiv \alpha / \sqrt{\kappa / 2}
$$

is the coupling parameter $\ell$ that we use to describe the axion-gravity coupling [26,32],

$$
\epsilon \equiv \frac{k-m_{a} / 2}{m_{a} / 2}
$$

is the fractional deviation of $k$ from the resonance frequency $m_{a} / 2$, and a useful dimensionless combination of parameters is

$$
\begin{aligned}
\gamma \equiv & \sqrt{2 \pi G} l^{2} m_{a}^{2} \frac{\left|a_{0}\right|}{2} \\
= & 5.7 \times 10^{-9} \\
& \times\left(\frac{\ell}{10^{8} \mathrm{~km}}\right)^{2}\left(\frac{m_{a}}{10^{-13} \mathrm{eV}}\right)\left(\frac{\rho_{a}}{0.3 \mathrm{GeV} / \mathrm{cm}^{3}}\right)^{1 / 2},
\end{aligned}
$$

where $m_{a}^{2}\left|a_{0}\right|^{2} / 2=\rho_{a}$ is used. For $m_{a}=10^{-13} \sim$ $10^{-10} \mathrm{eV}$ relevant to the LIGO band, $\gamma \ll 1$ for the most range of currently allowed coupling and density. Thus, the enhancement rate

$$
\mu \equiv \frac{m_{a}}{2} \sqrt{\gamma^{2}-\epsilon^{2}}
$$

will be assumed to be small throughout the paper. The origin of the name is clear from Eq. (6) which describes 
exponential enhancement when $\mu$ is real. These parameters will be used widely in our phenomenology study.

\section{B. Solution for finite propagation}

The solutions of the wave equation Eq. (6) can be expressed in terms of initial values $h_{F}^{(s)}(0)$ and $h_{B}^{(s)}(0)$ as

$$
\begin{aligned}
h_{F / B}^{(s)}(t)= & h_{F / B}^{(s)}(0) \cosh (\mu t) \\
& +\left[i \lambda^{(s)} \frac{\gamma e^{i \phi_{0}}}{\sqrt{\gamma^{2}-\epsilon^{2}}}\left(h_{B / F}^{(s)}(0)\right)^{*}\right. \\
& \left.-i \frac{\epsilon}{\sqrt{\gamma^{2}-\epsilon^{2}}} h_{F / B}^{(s)}(0)\right] \sinh (\mu t),
\end{aligned}
$$

where $\lambda^{(R / L)}=+1 /-1$ and $\phi_{0}$ denotes the phase part of the axion amplitude as $a_{0}=\left|a_{0}\right| e^{i \phi_{0}}$.

The initial condition relevant to the forward-propagating chirping GW is $h_{B}^{(s)}(0)=0$. Then, the solutions are

$$
\begin{gathered}
h_{F}^{(s)}(t)=h_{F}^{(s)}(0)\left[\cosh (\mu t)-i \frac{\epsilon}{\sqrt{\gamma^{2}-\epsilon^{2}}} \sinh (\mu t)\right] \\
h_{B}^{(s)}(t)=i \lambda^{(s)} \frac{\gamma e^{i \phi_{0}}}{\sqrt{\gamma^{2}-\epsilon^{2}}}\left(h_{F}^{(s)}(0)\right)^{*} \sinh (\mu t) .
\end{gathered}
$$

These GW solutions are of the same form as those of electromagnetic (EM) waves in $[17,23]$, even though the wave equations are different. These solutions are valid for both real and complex $\mu$ 's. We hereafter focus only on the forward wave as it is what we observe from binary mergers. This is overlooked in the previous GW work [26]; see Sec. VII for observational implications.

Now consider finite propagation of GW with $\mu t \ll 1$ in Eq. (13a), where $t$ is the propagation time. This limit will be relevant to the finite coherent axion patch. We first express Eq. (13a) in the polar form

$$
h_{F}^{(s)}(t)=h_{F}^{(s)}(0) \times F(t) \times e^{-i \psi(t)},
$$

and express $F(t)$ and $\psi(t)$ up to their lowest order axion contributions under $\mu t \ll 1(\gamma, \epsilon \ll 1$ is always assumed). They are

$$
\begin{aligned}
F(t) & \approx 1+\frac{\gamma^{2}}{2}\left(\frac{m_{a}}{2} t\right)^{2} \operatorname{sinc}^{2}\left(\frac{m_{a}}{2} \epsilon t\right) \\
& \equiv 1+\delta(\epsilon, t),
\end{aligned}
$$

where $\delta \ll 1$ by $\mu t \ll 1$ and

$$
\psi(t) \approx \frac{m_{a}}{2} \epsilon t\left\{1+\frac{1}{2}\left[\operatorname{sinc}\left(m_{a} \epsilon t\right)-1\right]\left(\frac{\gamma}{\epsilon}\right)^{2}\right\} .
$$

The leading term in the phase $\psi$, combined with the phase of the ansatz in Eq. (5), gives the phase velocity equal to the speed of light: $\left(m_{a} / 2\right) t+\left(m_{a} / 2\right) \epsilon t=k t$ so that $\omega=k$. Thus, the second term of Eq. (16) gives the correction to the dispersion relation as will be discussed in Sec. VC. Hereafter, we no longer distinguish the wave number $k$ and the angular frequency $\omega$ in the leading order. Similarly, for $F(t)$ in Eq. (15), the 1 refers to the original wave and the second term $\delta(\epsilon, t)$ is the enhancement due to the stimulated axion decay.

The resonance shape described by Eq. (15) is different from the naive expectation from Eq. (6). This is due to the finite propagation time, or equivalently the finite coherence of the axion field. In the next Sec. IV A, we discuss physical properties of these solutions with finite propagation time, in comparison to those with infinite propagation.

\section{SIGNAL}

We introduce two kinds of axion signals, main one in Sec. IVA and another in Sec. IV B. In the last two subsections, we discuss how to calculate them from the propagation through multiple coherent axion patches of a galactic halo.

\section{A. Signal 1: Resonance with finite coherence}

One may use the wave equation (6) to describe an exponential growth when $\mu$ is real for $\frac{m_{a}}{2}(1-\gamma)<k<$ $\frac{m_{a}}{2}(1+\gamma)$ from Eq. (11). The width $m_{a} \gamma$ is very narrow [see Eq. (10)] so that $k \approx m_{a} / 2$. This relation is consistent with the particle interpretation of the phenomenon as a stimulated axion decay into two gravitons. Thus, the growth is also called the "resonant enhancement." The maximum enhancement rate from Eq. (11) $\mu_{\max }=m_{a} \gamma / 2$ is also determined by $\gamma$.

An example of a resonance signal is shown in Fig. 1. As expected, the signal is indeed a sharp peak in the frequency spectrum of a chirping GW. However, the shape of the peak shown is not just determined by $m_{a}$ and $\gamma$ but modified by various effects that will be detailed now.

Above all, the finite coherence of the axion field makes important modifications. First, the resonance width is broadened, not simply given by $m_{a} \gamma$ as above. The resonance in each coherent patch is given by Eq. (15) with substituting $t$ by the coherent patch size $L_{\mathrm{coh}} \sim$ $1 / m_{a} \Delta v \sim 1 / m_{a} v(\Delta v \sim v$ is the velocity dispersion of axions; see Sec. IV C for more details),

$$
\begin{aligned}
F_{\text {patch }} & =1+\delta_{\text {patch }}(f) \\
& =1+\frac{\gamma^{2}}{2}\left(\frac{1}{2 \Delta v}\right)^{2} \operatorname{sinc}^{2}\left(\frac{\epsilon}{2 \Delta v}\right),
\end{aligned}
$$

where $\delta_{\text {patch }} \ll 1$ describes the enhancement at each patch. The frequency width of the enhancement is given by the central peak of the sinc function: $-2 \Delta v \lesssim \epsilon \lesssim 2 \Delta v$, 


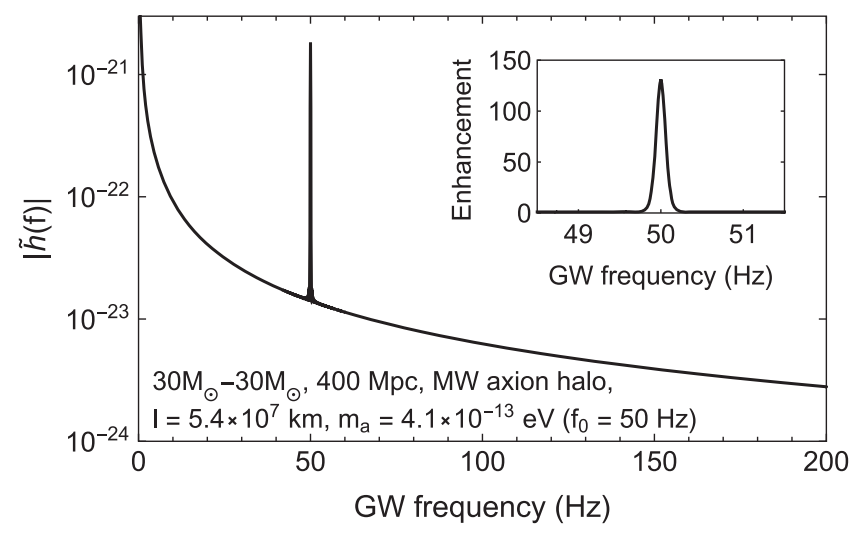

FIG. 1. An example axion signal in the chirping GW spectrum. The sharp peak is at the resonance frequency $f_{0}=50 \mathrm{~Hz}$ of the stimulated decay of axions corresponding to $m_{a}=$ $4.1 \times 10^{-13} \mathrm{eV}$. The inset shows the total enhancement from the propagation through a $100 \mathrm{kpc}$ axion halo. The coupling strength, $\ell=5.4 \times 10^{7} \mathrm{~km}$, is chosen to produce a barely detectable peak (Sec. VA and Fig. 2) showing that our detection criteria can be conservative. The effect of multiple coherent patches are maximally multiplied (Sec. IV D), and the resonance broadening is taken into account. The chirping GW is generated from $30-30 M_{\odot}$ binaries at $D=400 \mathrm{Mpc}$.

corresponding to $m_{a} / 2-m_{a} \Delta v \lesssim \omega_{\mathrm{GW}} \lesssim m_{a} / 2+m_{a} \Delta v$ yielding the peak width $\sim 2 m_{a} \Delta v$. This is different from the estimation above, where the width was determined by $\gamma$. The modification can be understood from two viewpoints. First, the length of the patch is $1 / m_{a} \Delta v$, so each patch cannot have a frequency resolution better than $m_{a} \Delta v$. This determines the resonance width. Another point of view is that axions have the velocity dispersion $\Delta v$, so that the observed resonance width is Doppler broadened by fractionally $\Delta v$. These two viewpoints are essentially the same, since the patch size is determined by the velocity dispersion.

The finite coherence also decreases the height of a peak, and the total height must be the sum of the enhancements from each coherent patch (since resonant enhancement occurs only within a coherent patch); this is discussed in Sec. IV D. All these effects are taken into account in Fig. 1. In our estimation, we will use this signal in the frequency domain, characterized by the peak frequency, height, and width.

However, the real LIGO data analysis will/must be done in both the frequency and time domains. This needs to account for the time duration and the time delay of the resonance. The time duration is $\sim 1 / m_{a} \Delta v$ which is related to the broadened frequency width $m_{a} \Delta v$ by the Fourier transform. This equals to the size of a coherent patch $L_{\mathrm{coh}}$, hence the time taken for a GW to pass one patch. And the time delay will be discussed in Sec. V C.

It is important to note that all these features of the resonance-frequency, width, amplification (height), time duration (temporal counterpart of frequency width), and time delay-must be almost the same among all observed GWs because all of them commonly pass through the MW axion halo. This allows the discovery of the resonance peak via correlations on these features. We will assume that the correlation can be made in the LIGO analysis, and focus on the above frequency-domain features of a single resonance in our numerical estimation. Instead, in Appendix B, we detail possible data analysis utilizing full features, and simulate the resulting signals in the frequency-time planespectrograms.

\section{B. Signal 2: Explosion}

Another constraint comes from the existence of certain dark matter substructures. If $\mu$ is too large, the stimulation becomes quicker and quicker so that a coherent axion patch becomes unstable and decays almost entirely into GWs. Such happens when [22]

$$
\mu_{\max } L_{\mathrm{coh}}=\frac{\gamma}{2 \Delta v}>1 \quad(\text { explosion }),
$$

which essentially means that the enhancement rate $\mu$ is larger than the passing time within a coherent patch $L_{\mathrm{coh}}=$ $1 / m_{a} \Delta v$ in Eq. (22). Thus, this may happen for small enough $\Delta v$ (long enough coherence) and high enough density $\rho_{a}$.

If there existed such substructures that could explode by satisfying Eq. (18), they must have almost disappeared by today because there are background photons and GWs everywhere with essentially any frequencies. Produced photons and GWs might have been dissipated enough or became a part of the stochastic backgrounds so that they might not be observable today. Instead, it is the observation of certain dark matter substructures surviving today which can impose an upper limit on the Chern-Simons coupling.

The observed dark matter substructures with possibly the largest enhancement rate are likely dwarf galaxies. They have small velocity dispersion $\Delta v=\mathcal{O}(1-10) \mathrm{km} / \mathrm{s} \simeq$ $10^{-5}$ and large dark matter density $\rho \simeq 10^{3} \times 0.3 \mathrm{GeV} / \mathrm{cm}^{3}$ at its central core [33] albeit some uncertainties. We conservatively use these values to estimate the upper bound on the Chern-Simons coupling, from the existence of dwarf galaxies; in any case, the bound on the coupling $\ell$ is not so sensitive to the density as it scales with $\rho_{a}^{1 / 4}$ in Eq. (10). As shown by the red solid in Fig. 2, this constraint is weaker than that from the resonance peak. Also, the approximation with small $\mu t \ll 1$ for the MW axion halo is thus good.

As an aside, which axion substructures could lead to explosion? The axion minicluster [34] has long coherence and high density. Virialized within its Jeans length $r_{J}$, the axion minicluster has [5]

$$
r_{J}=\frac{2 \pi}{\left(16 \pi G \rho_{\mathrm{mc}}\right)^{1 / 4} m_{a}^{1 / 2}} \sim \frac{1}{m_{a} \Delta v},
$$




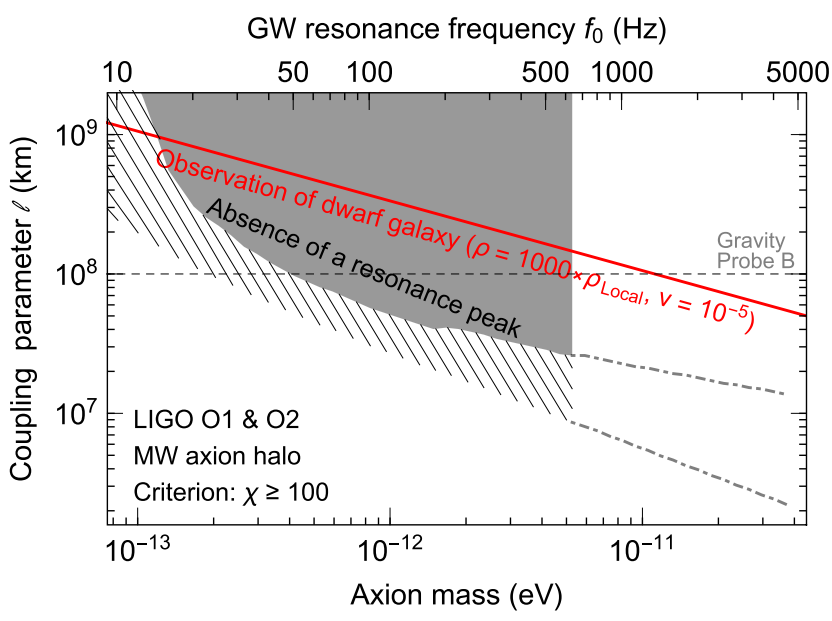

FIG. 2. The expected upper limit on the axion Chern-Simons coupling $\ell$, assuming the absence of resonance peaks in the $11 \mathrm{GW}$ observations at $\mathrm{LIGO} \mathrm{O} 1+\mathrm{O} 2$. The gray shaded region is excluded. The hatched band indicates ambiguities in summing up effects from multiple coherent patches in a halo (Sec. IV D). The dot-dashed extensions are the bounds that can be achieved with one more NS-NS observation (to be correlated with the one existing observation). The red solid is the bound from the existence of dwarf galaxies (Sec. IV B). The horizontal dashed is the established bound from the Gravity Probe B [8].

which is of order of the de Broglie wavelength. Here, $\rho_{\mathrm{mc}}$ is the density of a minicluster. Thus, the explosion $\mu_{\max } L_{\mathrm{coh}} \simeq$ $\mu_{\max } r_{J} \gtrsim 1$ [Eq. (18)] happens when

$$
\begin{aligned}
\ell \gtrsim & \left(7.3 \times 10^{6} \mathrm{~km}\right) \\
& \times\left(\frac{m_{a}}{10^{-12} \mathrm{eV}}\right)^{-3 / 4}\left(\frac{\rho_{\mathrm{mc}}}{0.3 \mathrm{GeV} / \mathrm{cm}^{3}}\right)^{-1 / 8} .
\end{aligned}
$$

As expected, this value of $\ell$ is much smaller than the bounds coming from dwarf galaxies and resonances (cf. Fig. 2). Although this estimate can be subject to small gravitational redshifts due to the minicluster itself and axion self interactions [23], we conclude that axion miniclusters are irrelevant to our work. If miniclusters had existed, they would have almost disappeared by today by explosion, or the axion coupling is too weak to be probed by any methods.

\section{Modeling an axion halo with multiple coherent patches}

A realistic axion halo is not infinitely coherent. The coherence property varies among axion dark substructures. As discussed in Sec. IV B, it is good enough to consider an axion halo without miniclusters; such a scenario is motivated by the misalignment production mechanism [35-37].

Such axion halo is virialized with the Milky Way (MW) whose total mass is $\sim 10^{12} M_{\odot}$ in a radius of $100 \mathrm{kpc}$. The virial velocity $v \sim 10^{-3}$ with the Maxwellian dispersion $\Delta v \sim v \sim 10^{-3}$ leads to the superposition of axion fields

$$
a(x, t)=a_{0} \cos (k x-\omega t+\phi)
$$

with the long spatial coherence length $1 / m_{a} \Delta v \sim 1 / m_{a} v \sim$ $10^{3} / m_{a}$ (with $k=m_{a} v$ ) [4]. This length is the size of a coherent patch

$$
L_{\mathrm{coh}}=\frac{1}{m_{a} \Delta v},
$$

in that a halo has a spatially oscillating profile with the oscillation length scale of $L_{\text {coh }}$. This is essentially the random-walk superposition of $N$ axion waves (with random phase) which leads to the total amplitude $\left|a_{0}\right| \propto \sqrt{N}$ consistent with the energy density given by $\rho_{a}=$ $m_{a}^{2}\left|a_{0}\right|^{2} / 2$ (see Eq. (25) for the value of $\rho_{a}$ ). In addition, $\omega=m_{a}\left(1+v^{2} / 2\right)$ so that the temporal coherence is broken only after a long time $1 / m_{a} \Delta v^{2} \sim 10^{6} / m_{a}$, much longer than the GW propagation time in each coherent patch. Thus, we ignore the temporal incoherence while taking into account the spatial incoherence.

Thus, an axion halo is composed of many smaller patches of sizes about the coherence length. As the GW propagates through an axion halo, it passes through multiple coherent patches. As the resonant effect grows only within a coherent patch, the total enhancement will be the sum of the individual patch's effect. We discuss how to sum them up in Sec. IV D.

In Sec. III, we have solved wave equations by assuming the spatially homogeneous and infinite axion field. We apply this solution to each coherent patch, which is actually of finite size and spatially varying. In effect for simplicity, we approximate each coherent patch as a Heaviside profile with the length $L_{\mathrm{coh}}$ and the amplitude satisfying $\rho_{a}=m_{a}^{2}\left|a_{0}\right|^{2} / 2$. The solution is thus good enough well inside the patch, but our calculation does not include the entrance and exit of GWs through the boundary of a patch. Nevertheless, this approximation can still capture the main physics of the phenomenon. We defer more accurate calculations to the future.

\section{Summing effects from multiple patches}

A dark matter halo in a galaxy consists of many smaller coherent patches. The resonant enhancement occurs only within a coherent patch. Therefore, we need to sum the enhancement from each patch to get the total effect coming from propagation through an axion halo.

There is a subtlety here. As discussed in Sec. IV A, the resonance has a time duration of $L_{\mathrm{coh}}$ due to the finite frequency width of a resonance. Thus, not all resonance stimulates axion decays simultaneously. It is complicated to account for the fraction of GWs participating in the stimulation at each moment. But we know that the original chirping GW is the largest and dominantly stimulates the axion decay at early time of propagation. At later times, the 
enhanced signal grows larger than the original chirping one, and the issue becomes more relevant.

Rather than figuring out an accurate method, we estimate the range of the maximum and minimum possible summation. The enhancement in one patch is $1+\delta(f)$ from Eq. (15) [and Eq. (17)], where $\delta \ll 1$ is peaked at the central resonance frequency $f_{0}$. What is the enhancement after passing $N$ patches? The maximum summation assumes that all the axion signals from one patch contribute to the stimulation in the next patch, yielding the maximum total enhancement

$$
(1+\delta(f))^{N} \approx e^{N \delta(f)} \quad(\text { maximum sum }) .
$$

On the other hand, the minimum summation assumes no axion signals but only original GW stimulates in the next patch. This yields the minimum total enhancement

$$
1+N \delta(f) \quad \text { (minimum sum) }
$$

We use these two estimations to obtain an uncertainty band of our estimation (see Fig. 2, for example). A more realistic summation is likely to be between them.

In both cases, the summation depends on the $N \delta(f)$. As $\delta$ depends linearly on $\rho\left(\delta \propto \gamma^{2} \propto \rho\right)$, we can use the lineaveraged density along the line of sight (LOS) for each coherent patch. For a LOS toward outside the galactic halo, the following line-averaged density is obtained

$$
\bar{\rho}_{\mathrm{LOS}}=\frac{\int_{8 \mathrm{kpc}}^{100 \mathrm{kpc}} \rho(r) d r}{(100 \mathrm{kpc}-8 \mathrm{kpc})} \approx 0.04 \mathrm{GeV} / \mathrm{cm}^{3}
$$

for both NFW and Burkert profiles $\rho(r)$ of the MW dark matter halo, where $100 \mathrm{kpc}$ is the assumed halo radius and $8 \mathrm{kpc}$ is our distance from the MW center. We have taken best-fit parameters for both profiles from [38]. We have checked that $\int_{8 \mathrm{kpc}}^{100 \mathrm{kpc}} \rho(r) d r \simeq 0.98 \int_{8 \mathrm{kpc}}^{\infty} \rho(r) d r$ for both profiles, confirming that the $100 \mathrm{kpc}$ radius is sufficient. We use this average value for $\rho_{a}$ in our numerical study.

Last, the factor $N \delta$ makes the importance of finite coherence in yet another manifest way. From Eq. (9) and Eq. (17), we have $\delta_{\text {patch }}(f) \propto \rho \ell^{4} m_{a}^{2} / \Delta v^{2} \times$ $\operatorname{sinc}^{2}(\epsilon / 2 \Delta v)$. For the travel through the MW axion halo of size $R$, there are $N=R m_{a} \Delta v$ number of coherent patches. So the whole enhancement depends on the combination

$$
(N \delta(f))_{\text {halo }} \propto \frac{R \rho_{a} \ell^{4} m_{a}^{3}}{\Delta v} \times \operatorname{sinc}^{2}\left(\frac{\epsilon}{2 \Delta v}\right)
$$

The linear dependence on the $R$ and $\rho$ is reasonable, and the overall dependence on $1 / \Delta v$ implies that the enhancement is greater for a longer coherence from a smaller velocity dispersion. Thus, the effect of finite coherence indeed suppresses the size of the enhancement while broadening the frequency width.

\section{LIGO BOUNDS AND PROSPECTS}

We use the properties (chirp mass and luminosity distance $)^{4}$ of $11 \mathrm{GW}$ events in LIGO $\mathrm{O} 1$ and $\mathrm{O} 2$ to estimate the potential constraints on the axion coupling. We use the leading-order quadrupole waveform up to the innermost stable circular orbit, which is a good approximation for the 11 LIGO events. As discussed in Sec. IVA, we use the signal features in the frequency domain in the estimation, while postponing the discussion of a more realistic analysis utilizing the full features in Appendix B.

\section{A. Detection criteria}

We measure the likelihood $-2 \ln L$ of the existence of a resonance peak using the peak strength as

$$
-2 \ln L=\chi^{2} \equiv \sum_{i}\left(\Delta \mathrm{SNR}_{\text {peak }, i}\right)^{2},
$$

where $i$ is summed over all observed GWs. The peak strength $\Delta \mathrm{SNR}_{\text {peak }}$ is defined as the SNR in the resonance region $[-2 \pi \Delta v \leq \epsilon \leq 2 \pi \Delta v$ from Eq. (17)] subtracted by the SNR of the original chirping GW in the same frequency range; this roughly measures the significance of the deviation from smooth chirping. The $\Delta \mathrm{SNR}_{\text {peak }}$ can be calculated from Eq. (14) or by multiplying Eq. (23) or Eq. (24) to the original waveform.

For detection, the likelihood is required to be larger than 100:

$$
\chi \geq 100 \quad \text { (detection criteria). }
$$

This is the only requirement in our numerical estimation assuming that the correlation of all observed GWs can be well made. ${ }^{5}$ It is strong enough. First, the $\Delta \mathrm{SNR}_{\text {peak }}=100$ is much larger than the usual detection threshold of 8-10. Further, such strong signal is well above the fractional uncertainty of the amplitude measurement $\sim 1$ SSR [39], which is $\sim \mathcal{O}(10) \%$ for the 11 LIGO events with SNR $\mathcal{O}(1)$ in the resonance region. Figure 1 and Fig. 4 show that signals satisfying the criteria are indeed sharp and large.

Nevertheless, the criteria is not loosened as real analysis including matched filtering and correlation may bring additional uncertainties. Also, such estimation can be good enough because the signal strength $\Delta \mathrm{SNR}_{\text {peak }}$ depends on $\ell$

\footnotetext{
${ }^{4}$ For simplicity, we assume $h=h_{+} F_{+}$polarization with orientation $\mathbf{L} \cdot \mathbf{n}=0$ and antenna projection $F_{+}=1$. Since the LIGO measurements are short, actual measurements will only be multiplied by a constant factor.

${ }^{5}$ This simple requirement may be mimicked by a strong accidental noise in a single GW event, but the correlation of all GWs may reduce the likelihood of such a fake.
} 
strongly [N $\delta \propto \gamma^{2} \propto \ell^{4}$ from Eq. (26)] so that mild loosening of the criteria does not bring large improvement on the $\ell$ bound.

\section{B. Results}

In Fig. 2, we show the expected LIGO bound on the axion Chern-Simons coupling $\ell$ as a function of the axion mass $m_{a}$ (the corresponding peak GW frequency $f_{0}$ is shown on the upper horizontal axis). The gray shaded region can be excluded, from the assumed absence of resonance peaks in the 11 LIGO observations so far (Signal 1 in Sec. IVA); each GW is considered up to its innermost stable circular orbit. This region is obtained by the most pessimistic summation of multi-patch effects as in Eq. (24). The hatched region indicates ambiguities in the summation method; this is the region that could be excluded if a somewhat more optimistic summation can be used. This region extends to the lower range of $\ell$ obtained by the most optimistic summation in Eq. (23). A more realistic bound may lie somewhere in this band (Sec. IV D). The dot-dashed extension of the bounds are the expected bounds with one more NS-NS observation so that a correlation can be made with existing NS-NS data in the highest frequency range $\mathcal{O}(1000) \mathrm{Hz}$; heavier binaries merge at lower frequencies. The existing bound from Gravity Probe B satellite measurement of the frame dragging effect [8] is shown as the horizontal dashed. The bound from the existence of dwarf galaxies (imposing that such systems are not exploded by resonant enhancement) is shown as the red solid (Signal 2 in Sec. IV B).

The LIGO bound from the absence of a peak is stronger than the existing established bound, at least for the axion mass range $5 \times 10^{-13} \mathrm{eV} \lesssim m_{a} \lesssim 5 \times 10^{-12} \mathrm{eV}$. The bound can be stronger if a more aggressive summation can be used, and the heavier mass range up to $m_{a} \simeq 5 \times$ $10^{-11} \mathrm{eV}$ can be constrained if more NS-NS mergers (to be correlated) are observed.

How will the bound improve with more data and smaller noise? For example, a 10 times smaller noise (achievable with, e.g., Einstein Telescope) will enhance SNR by 10 and observe $10^{3}$ times more GWs, yielding $\approx(10 \times \sqrt{1000})^{1 / 4} \simeq 4.2$ times stronger bound on $\ell$. Similarly, $n$ times smaller requirement on $\chi$ means $n^{1 / 4}$ times stronger bound on $\ell$. The measurement of lower frequency range from future $\mathrm{GW}$ detectors can also provide new constraints on the lower range of $m_{a}$.

One can also note that the bound becomes stronger for the heavier axion. This is basically because $\gamma \propto m_{a}$ for a given axion energy density $\rho_{a}$ [see Eq. (10)], giving $(N \delta)_{\text {halo }} \propto m_{a}^{3}$ as in Eq. (26). This strong dependence on $m_{a}$ overcomes the frequency dependences of the noise curve and chirping GW spectrum; but additional slight mass dependence of the bound comes from these.

In all, LIGO can potentially obtain the strongest bounds on the axion-gravity Chern-Simons coupling, at least for $m_{a}=5 \times 10^{-13}-5 \times 10^{-12} \mathrm{eV}$. We encourage a careful reanalysis of the currently available data.

\section{Time-delay of a resonance from dispersion}

The resonant enhancement also modifies the group velocity of a resonance peak, delaying the arrival of the peak relative to the original chirping part. This does not appear in frequency-domain waveforms but only in time domain ones. Any full data analysis must account for this time delay.

The dispersion relation can be obtained from the correction term in Eq. (16) and ansatz (5) for the case of small enhancement $(\mu t \ll 1)$ in the vicinity of $\epsilon=0$ as

$$
\omega(k)=k-\left(k-\frac{m_{a}}{2}\right)\left(\frac{m_{a}}{2} \gamma t\right)^{2} .
$$

This means that $\omega=k$ at $t=0$ (not enhanced yet) but starts to deviate from $k$ as GW propagates through a coherent patch. Note that the dispersion is parity independent; see Sec. VII for usual parity-dependent dispersion. For $k>m_{a} / 2, \omega$ decreases from $k$ toward $m_{a} / 2$, and opposite for $k<m_{a} / 2$. As $\mu t$ grows larger than 1 , referring back to more general equation Eq. (13a), we find that the phase converges to some constant which implies [by ansatz Eq. (5)] $\omega=m_{a} / 2$ regardless of $k$. This behavior is approximately understood because the $\mathrm{GW}$ produced from axion decays has $\omega=m_{a} / 2$ by the energy conservation, while its spatial mode is determined by initial chirping GW with the wave number $k \neq m_{a} / 2$. As the enhancement grows, $\omega=m_{a} / 2=k$ dominates a whole $\mathrm{GW}$.

Back to Eq. (29) with $\mu t \ll 1$, the group velocity of the axion signal $v_{g}=[d \omega / d k]_{\epsilon=0}$ is

$$
v_{g}=1-\left(\frac{m_{a}}{2} \gamma t\right)^{2} \text {. }
$$

This again means that $\omega=k$ and $v_{g}=1$ at $t=0$ (not enhanced yet) starts to deviate with $t$. The group velocity is less than 1 so that the axion signal arrives later than the chirping part.

Averaged over the time dependence along the propagation through a coherent patch (this is also the average group velocity in the galactic axion field), the average group velocity is given by

$$
\bar{v}_{g}=1-\frac{1}{3}\left(\frac{\gamma}{2 \Delta v}\right)^{2} .
$$

With $100 \mathrm{kpc}$ halo size, Eq. (31) gives the time delay of the resonance peak with respect to the chirping GW. In Fig. 3, we plot contours of this time delay. In the parameter space that can be probed at LIGO, the time delay is $1-100 \mathrm{sec}-$ onds, which is also about the duration of a resonance $\sim 1 / m_{a} \Delta v=1-100$ seconds. This timescale is longer than 


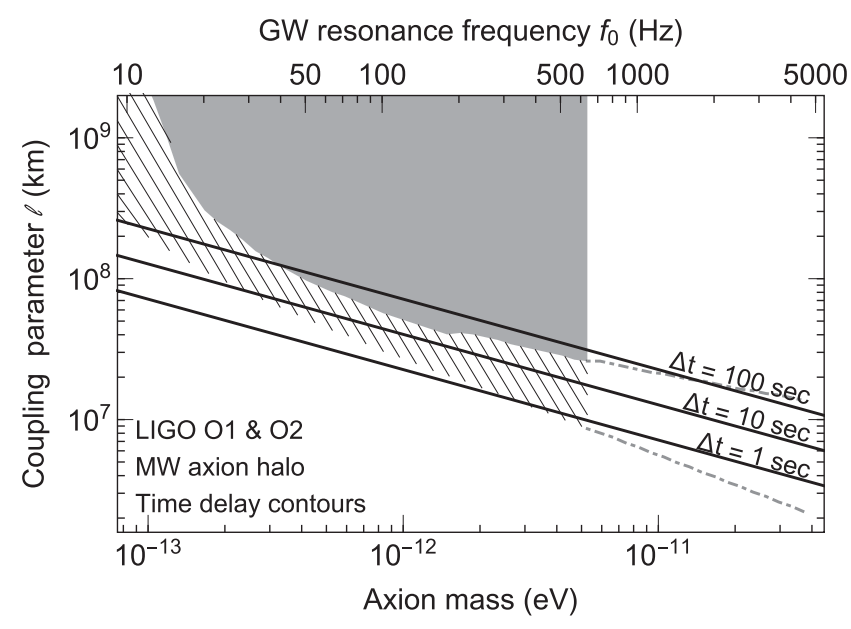

FIG. 3. The contours (solid) of the arrival-time delay of a resonance peak with respect to the chirping part, induced from the propagation through a $100 \mathrm{kpc}$ axion halo. The overlaid are the exclusion plots in Fig. 2.

the typical duration (seconds or less) of chirping GWs in the LIGO band. This could complicate the search because the temporal separation of axion signals and the chirping part may conceal the association between them. Thus, realistic data analysis needs to test longer time series of data than the chirping itself. Such an analysis accounting for this feature will be discussed in Appendix B with resulting spectrograms in Fig. 4.

\section{Similar bounds on the axion-photon coupling}

We briefly comment on the axion-photon coupling. Since the solution of the coupled EM wave equations is in the same form as Eq. (13a) [17,23], we can readily apply the same analysis done here to the photon case. The signal would be the extragalactic EM waves with a common peak. By simply requiring the maximum total enhancement of a single good EM signal to be greater than 10 (as light measurements are more precise), we estimate the bound on the axion-photon coupling to be $g_{\text {ary }} \lesssim 10^{-8}-10^{-2} \mathrm{GeV}^{-1}$ for the axion mass range $10^{-11}-1 \mathrm{eV}$. This is similar or slightly weaker than the laboratory bounds, while much weaker than the Helioscope bound by about $2-8$ orders of magnitudes [40]. We defer more detailed analysis and comparison to a future project.

\section{DISCUSSIONS}

\section{A. Energy conservation and axion backreaction}

The energy conservation implies that the amplitude of the axion field should decrease as the GW gets amplified. However, dark matter energy density is much greater than any reasonable GW energy density. We can estimate the chirping GW energy density as the following. GW150914 emitted $\sim 3 M_{\odot}$ of energy at $400 \mathrm{Mpc}$, and by assuming all the energy was released in the last 0.1 second of chirping, we have $\rho_{\mathrm{GW}}=60 \mathrm{eV} / \mathrm{cm}^{3}$. This is incomparably smaller than the dark matter energy density $\bar{\rho}_{\mathrm{DM}}=0.04 \mathrm{GeV} / \mathrm{cm}^{3}$ in Eq. (25). Thus, we can assume that axion fields do not decrease in our work.

But if somehow energies of both waves become similar, the coupled wave equations describe the energy transfer between them through the time evolution of both amplitudes. For example for the EM wave case, Eq. (8) and Fig. 2 of [17] show such time variation. Back to a general point of view, the absence of explicit time dependence of the Lagrangian guarantees the energy conservation for a dynamically-evolving axion field. After all, the backreaction of axion fields will stop the exponential growth of GWs at some point.

\section{B. Stimulated axion decay rate}

We obtain another insight on the stimulated decay by calculating the axion decay rate from the energy gain of the $\mathrm{GW}$, which equals to the energy loss of the axion. Since the spatially averaged energy density of $\mathrm{GW}$ is given by $\left\langle\rho_{\mathrm{GW}}\right\rangle=\omega_{\mathrm{GW}}^{2}\left(\left|\tilde{h}^{(R)}\right|^{2}+\left|\tilde{h}^{(L)}\right|^{2}\right) / 64 \pi G$, the energy density gain of the forward and backward waves are

$$
\begin{aligned}
\Delta\left\langle\rho_{\mathrm{GW}}\right\rangle_{F} & =\left\langle\rho_{\mathrm{GW}}\right\rangle_{0}\left(F^{2}(t)-1\right) \\
& \approx\left\langle\rho_{\mathrm{GW}}\right\rangle_{0} \times \gamma^{2}\left(\frac{m_{a}}{2}\right)^{2} \frac{\sin ^{2}\left[\left(\omega_{\mathrm{GW}}-\frac{m_{a}}{2}\right) t\right]}{\left(\omega_{\mathrm{GW}}-\frac{m_{a}}{2}\right)^{2}} \\
& =\Delta\left\langle\rho_{\mathrm{GW}}\right\rangle_{B},
\end{aligned}
$$

where the last equality is due to the momentum conservation [this can also be explicitly derived from Eq. (13b)].

From the energy density loss of the axion $2 \times \Delta\left\langle\rho_{\mathrm{GW}}\right\rangle_{F}$, we obtain the decay rate of the axion as (for $\mu t \ll 1$; otherwise, the rate increases exponentially)

$$
\begin{aligned}
P_{\text {decay }}(t) & =\frac{-\Delta \rho_{a}}{\rho_{a}} \\
& =\left\langle\rho_{\mathrm{GW}}\right\rangle_{0} \times \frac{1}{2} \pi G l^{4} m_{a}^{4} \frac{\sin ^{2}\left[\left(\omega_{\mathrm{GW}}-\frac{m_{a}}{2}\right) t\right]}{\left(\omega_{\mathrm{GW}}-\frac{m_{a}}{2}\right)^{2}} .
\end{aligned}
$$

As it should be, this is proportional to the energy density of the GW and independent on the axion energy density. Remarkably, the form of $\sin ^{2}\left(\left(\omega-\omega_{0}\right) t\right) /\left(\omega-\omega_{0}\right)^{2}$ is nearly identical to the probability of stimulated emission in quantum mechanics [41]. This consideration supports the physical picture of the resonant enhancement as the stimulated decay.

\section{Effective "graviton" mass}

Even without the axion-gravity Chern-Simons coupling, the GW experiences a dispersion due to the intervening mass density, similarly to the photon's plasma mass in the electron medium. Following the EM wave case in [22], we check that such effect is negligible for the GW. 
The GW refractive index is given by $n=1+2 \pi G \rho / \omega^{2}$ [42]. This gives the dispersion relation $\omega^{2}=k^{2}-4 \pi G \rho$. The ratio of the matter-induced dispersion to the effect of gravitational Chern-Simons coupling in Eq. (A4) is

$\frac{4 \pi G \rho}{m^{2} \gamma}=6.3 \times 10^{-50} \times \sqrt{\frac{\rho}{\mathrm{GeV} / \mathrm{cm}^{3}}} \times\left(\frac{m}{\mathrm{eV}}\right)^{-3} \times\left(\frac{\ell}{\mathrm{km}}\right)^{-2}$

at the resonance. This is an incredibly small number; for instance, $\rho=1 \mathrm{GeV} / \mathrm{cm}^{3}, \quad m=10^{-12} \mathrm{eV}$, and $\ell=10^{8} \mathrm{~km}$ give $6.3 \times 10^{-30}$. Thus, the dispersion of GWs due to intergalactic matter can be ignored.

\section{Axions in the source galaxy and intergalactic region}

The sharp peak was unambiguously identified as the axion signal because every GW will exhibit a common peak from the propagation through the MW axion halo. What about axions in other galaxies (in particular, host galaxies of GW sources) and intergalactic region?

First, the axions in the host galaxy will also undergo similar stimulated decays. However, when observed at LIGO, their resonance frequency is redshifted from the cosmological expansion, hence different from that of the MW halo. As the redshift of the host galaxy varies among GW events, the resonance signals cannot be correlated neither. Thus, they presumably do not affect our study. But besides this, strong GWs near a source may significantly alter the local axion field, or the existence of axions might affect the dynamics of the GW production (one simple example is [15]).

As for intergalactic axions, its effect may not be strong enough due to low density and varying redshift. While a half of total dark matter resides in the intergalactic region, its density $\Omega_{\mathrm{DM}} \rho_{c} \sim 10^{-6} \mathrm{GeV} / \mathrm{cm}^{3}$ is $10^{-4}-10^{-5}$ times smaller than $\bar{\rho}_{a}$ in the MW halo [Eq. (25)]. Thus, the enhancement $N \delta \propto R \rho_{a} / v$ [Eq. (26)] is proportionally smaller. This may be compensated by either long propagation more than a few Gpc $\left(10^{4}-10^{5}\right.$ times longer than the MW halo size) or much smaller velocity dispersion. But the former with continuously varying redshift through intergalactic region further hinders the generation of sharp and large signals. The latter is unlikely because the Local Group velocity with respect to the $\mathrm{CMB}$ and the escape velocity of the MW are all $\mathcal{O}(100) \mathrm{km} / \mathrm{s}$. Thus, intergalactic contributions are ignored.

\section{COROLLARY: ABSENCE OF PARITY-VIOLATION OBSERVABLES ON THE CHIRPING GW}

Many previous works have studied the parity-violation signals in photons due to the axion Chern-Simons coupling. However, our solution shows that the parity-violation is not observable in the resonance regime of the chirping $\mathrm{GW}$. They are not in contradiction as we explain in this section.

The parity violation in previous works are manifest in two ways: one is through the dispersion relation $[16,28$, 43-47] and the other through the enhancement [16,26]. For our case, the parity-dependent dispersion is absent because we consider the resonance regime $\left(k \approx m_{a} / 2\right)$, and the paritydependent enhancement is absent because we consider the forward propagation of waves (not stochastic waves).

First, the parity-dependent dispersion is obtained from the wave equation in the form of Eq. (A4) as

$$
\omega(k)^{2}=k^{2}+2 \lambda^{(s)} m_{a} k \gamma \sin \left(m_{a} t\right) .
$$

This shows the usual parity-dependent $\left(\lambda^{(s)}\right.$-dependent) dispersion relation, making the phase velocity deviate from $c$ and oscillate oppositely for opposite polarizations. But the deviation (the second term) oscillates in time with a frequency $1 / m_{a}$. In previous works with $k \gg m_{a} / 2$, this oscillation was much slower than the high frequency of photons. But in our case in the resonance regime, they are comparable $\left(k \approx m_{a} / 2\right)$ so that the deviation almost averages out in one period of the GW/photon. Instead, near the resonance, there arises the dispersion relation which is parity independent, as discussed in Sec. V C.

Second, the usual parity-dependent enhancement arises from the time evolution of spatial Fourier modes, $\tilde{h}(k, t)=$ $\int h(x, t) e^{-i k x}[16,26]$. But these spatial modes are the sum of forward and backward propagating waves. By separating the propagation direction, as in our solution Eq. (13a) and (13b), we find that the parity-violation exists only in backward waves due to the initial condition $h_{B}=0$. This effect is not observable in our case because what we observe is only $h_{F}$. The previous solutions are suitable for stochastic backgrounds, like CMB or stochastic GWs, where waves with all directions are mixed up. Such waves can exhibit the parity violation as polarization-dependent enhancements, as studied in previous works.

\section{CONCLUSIONS}

We have shown that the LIGO observation of chirping GWs can constrain the axion-gravity Chern-Simons coupling, through a resonance peak of the GW induced by the coherently oscillating axion dark matter field. As all the observed GWs pass through the MW axion halo, they will have a peak with common properties of frequency, amplification, frequency width or time duration, and time delay. The correlation among them will confidently detect or reject the peak. We have found that $11 \mathrm{GW}$ observations at LIGO $\mathrm{O} 1+\mathrm{O} 2$ can potentially provide the strongest bound on the coupling, at least for $m_{a}=5 \times 10^{-13}-5 \times 10^{-12} \mathrm{eV}$ (Fig. 2). With more LIGO observations, the range can be extended and the bound can be stronger. A careful reanalysis of existing data is encouraged. 
The finite coherence of the axion field largely affects the resonance (hence the axion signal) shape. First, it suppresses the height and broadens the width of the resonance peak in the frequency spectrum of the GW. The broadening in the frequency domain also makes the signal persist as long as the size of a coherent patch. The resonanceproduced axion signal is also time delayed compared to the original chirping part, where the delay is also affected by the finite coherence. Dedicated resonance searches must account for all these effects.

The resonance phenomenon is essentially the stimulated decay of the axion. Not only does the resonance condition $\omega_{0} \simeq m_{a} / 2\left(f_{0} \simeq m_{a} / 4 \pi\right)$ support this particlelike interpretation, but also the decay probability estimated from the energy gain and loss of the fields agrees with the quantum mechanical description of stimulated emissions and absorptions. This is remarkable as we have never quantized these waves.

A proper ansatz treating forward and backward-going waves separately is crucial for our work. It is because only forward-going waves are observed while backward-going waves are also necessarily produced by momentum conservation. The observational situation is different from the stochastic background of GWs and CMB, where waves with all directions are mixed up. As a consequence, our solution does not exhibit the parity violation from the axion Chern-Simons coupling in the forward-going wave at the linear order, but this is not in contradiction with previous studies of parity-violation observables.

Last but not the least, the resonant effect can sometimes become so efficient that an axion substructure may not exist today. This happens when the axion structure has small velocity dispersion (hence, long coherence) and high density. The axion minicluster is one example that might have exploded by today, but the coherent axion field virialized with a whole galaxy does not explode given the current bound on the coupling. Although we assumed that the signal of explosion had diffused away, it would be interesting to study if any observable signals remain.

In all, we have studied one way to probe and constrain the axion-gravity Chern-Simons coupling, which is generic and well motivated. A careful reanalysis of LIGO data may provide one of the strongest constraints on this coupling. Various other types of axion-gravity couplings may also be probed in a similar way.

\section{ACKNOWLEDGMENTS}

Authors would like to thank Han Gil Choi, Kwang Sik Jeong, Hyung Do Kim, Hyungjin Kim, Ji-hoon Kim, Chang Sub Shin, Takahiro Tanaka, Eungwang Seo, Tjonnie G. F. Li for useful discussions. S. J. and T. H. K. are supported by the NRF of Korea under Grants No. NRF2019R1C1C1010050, No. 2015R1A4A1042542 and S. J. also by POSCO Science Fellowship. J. S. is supported in part by JSPS KAKENHI Grants No. JP17H02894,
No. JP17K18778, No. JP15H05895, No. JP17H06359, No. JP18H04589. Y. U. is supported by Grant-in-Aid for Scientific Research on Innovative Areas under Contract No. 18H04349, Grant-in-Aid for Scientific Research (B) under Contract No. 19H01894, and the Deutsche Forschungsgemeinschaft (DFG, German Research Foundation)—Project No. 315477589-TRR 211. J. S. and Y.U. were also supported by JSPS Bilateral Joint Research Projects (JSPS-NRF collaboration) "String Axion Cosmology" and benefitted from discussions during the YITP workshop YITP-T-19-02 on "Resonant instabilities in cosmology". The research of S.J. and Y.U. was supported in part by the National Science Foundation under Grant No. NSF PHY-1748958.

\section{APPENDIX A: SOLVING WAVE EQUATION THROUGH THE MATHIEU EQUATION}

In this Appendix, we solve Eq. (2) by another method. By expressing $h_{i j}$ in Eq. (2) as a Fourier transform, the wave equation for each Fourier amplitude becomes [26]

$$
\ddot{\tilde{h}}^{(s)}+\frac{4 \lambda^{(s)} \gamma \cos \left(m_{a} t\right)}{1+4 \lambda^{(s)} \frac{k}{m_{a}} \gamma \sin \left(m_{a} t\right)} k \dot{\tilde{h}}^{(s)}+k^{2} \tilde{h}^{(s)}=0,
$$

where $\tilde{h}^{(s)}$ represents the spatial Fourier mode with wave number $k$ (so different from the amplitude $h_{F / B}^{(s)}$ appearing in Eq. (5); $\tilde{h}^{(s)}$ contains the full oscillation part $e^{-i \omega t}$ ). Since we only want to see the leading order, we rewrite the equation as

$$
\ddot{\tilde{h}}^{(s)}+4 \lambda^{(s)} \gamma \cos \left(m_{a} t\right) k \dot{\tilde{h}}^{(s)}+k^{2} \tilde{h}^{(s)}=0 .
$$

By following the transformation in [28] with cosmic expansion neglected, we define $\psi$ as

$$
\tilde{h}^{(s)}=e^{-2 \int \lambda^{(s)} \gamma k \cos \left(m_{a} t\right) d t} \Psi,
$$

and we have

$$
\ddot{\Psi}+\left(k^{2}+2 \lambda^{(s)} m_{a} k \gamma \sin \left(m_{a} t\right)\right) \Psi=0
$$

which is the ordinary Mathieu equation. The exponential factor in Eq. (A3) is a nonresonant term since the exponent oscillate with small amplitude. Any analytic method solving the Mathieu equation tracks only the resonant term, so this factor does not affect the result. Also physically, this factor will be canceled out in average, due to its dependence on the relative phase between the axion field and the gravitational wave (for such cases, the argument of cos's and sin's should have a constant phase term, like $\left.m t+\psi_{0}\right)$.

To solve Eq. (A4) by the two variable expansion method $[48,49]$ we look at the solution behavior near the resonance 
at $k=m_{a} / 2$. For this, we use $(4 \gamma)$ for an expansion parameter, and the expansion will be done up to the first order. The two variables in the expansion are the ordinary time $\xi=t$ and the slow time $\eta=4 \gamma t$. $\xi$ is the time scale of wave oscillation, while $\eta$ is the time scale of amplitude change. We regard $\Psi$ as a function of the two independent variables, as $\Psi=\Psi(\xi, \eta)$. And the time derivative operator becomes

$$
\frac{d}{d t}=\frac{\partial}{\partial \xi}+4 \gamma \frac{\partial}{\partial \eta} .
$$

Similarily, $k$ and $\Psi$ are expanded as

$$
k=\frac{m}{2}\left[1+\epsilon_{1}(4 \gamma)+\epsilon_{2}(4 \gamma)^{2}+\cdots\right]
$$

and

$\Psi(\xi, \eta)=\Psi_{0}(\xi, \eta)+(4 \gamma) \Psi_{1}(\xi, \eta)+(4 \gamma)^{2} \Psi_{2}(\xi, \eta)+\cdots$.

By putting Eqs. (A5)-(A7) into Eq. (A4), we can obtain series of equations assorted by the order in $(4 \gamma)$. The 0th order equation is

$$
\frac{\partial^{2} \Psi_{0}}{\partial \xi^{2}}+\left(\frac{m_{a}}{2}\right)^{2} \Psi_{0}=0,
$$

and the 1 st order equation is (note that $\xi=t$ )

$$
\begin{aligned}
& \frac{\partial^{2} \Psi_{1}}{\partial \xi^{2}}+\left(\frac{m_{a}}{2}\right)^{2} \Psi_{1} \\
& =-2 \frac{\partial^{2} \Psi_{0}}{\partial \xi \partial \eta}-\lambda^{(s)} \frac{m_{a}^{2}}{4} \sin \left(m_{a} \xi\right) \Psi_{0}-\frac{m_{a}^{2}}{2} \epsilon_{1} \Psi_{0} .
\end{aligned}
$$

Eq. (A8) gives $\Psi_{0}$ in the form of

$$
\Psi_{0}=A(\eta) \cos \left(\frac{m_{a}}{2} \xi\right)+B(\eta) \sin \left(\frac{m_{a}}{2} \xi\right),
$$

and putting this into Eq. (A9) with using trigonometric identities gives

$$
\begin{aligned}
& \frac{\partial^{2} \Psi_{1}}{\partial \xi^{2}}+\left(\frac{m_{a}}{2}\right)^{2} \Psi_{1} \\
& =\left(m_{a} A^{\prime}(\eta)-\lambda^{(s)} \frac{m_{a}^{2}}{8} A(\eta)-\frac{m_{a}^{2}}{2} \epsilon_{1} B(\eta)\right) \sin \left(\frac{m_{a}}{2} \xi\right) \\
& \quad+\left(-m_{a} B^{\prime}(\eta)-\lambda^{(s)} \frac{m_{a}^{2}}{8} B(\eta)-\frac{m_{a}^{2}}{2} \epsilon_{1} A(\eta)\right) \cos \left(\frac{m_{a}}{2} \xi\right) \\
& \quad+\cdots
\end{aligned}
$$

where the higher frequency terms in the right-hand side whose resonance appear only in higher orders are not shown. This gives the slow flow equations for $A$ and $B$ as

$$
\frac{d}{d \eta}\left(\begin{array}{l}
A(\eta) \\
B(\eta)
\end{array}\right)=\left(\begin{array}{cc}
\lambda^{(s)} \frac{m_{a}}{8} & \frac{m_{a}}{2} \epsilon_{1} \\
-\frac{m_{a}}{2} \epsilon_{1} & -\lambda^{(s)} \frac{m_{a}}{8}
\end{array}\right)\left(\begin{array}{c}
A(\eta) \\
B(\eta)
\end{array}\right) .
$$

We find a solution in the form of

$$
\left(\begin{array}{l}
A(\eta) \\
B(\eta)
\end{array}\right)=\left(\begin{array}{l}
a \\
b
\end{array}\right) e^{\nu \eta}
$$

By putting Eq. (A13) into Eq. (A12), we get

$$
\nu= \pm \frac{m_{a}}{8} \sqrt{1-16 \epsilon_{1}^{2}}
$$

and

$$
\begin{aligned}
& \left(\begin{array}{l}
a \\
b
\end{array}\right)_{+}=\left(\begin{array}{c}
1 \\
-\frac{\lambda^{(s)}-\sqrt{1-16 \epsilon_{1}^{2}}}{4 \epsilon_{1}}
\end{array}\right), \\
& \left(\begin{array}{l}
a \\
b
\end{array}\right)_{-}=\left(\begin{array}{c}
-\frac{\lambda^{(s)}-\sqrt{1-16 \epsilon_{1}^{2}}}{4 \epsilon_{1}} \\
1
\end{array}\right) .
\end{aligned}
$$

We put these into Eq. (A10) and recover the notations we used in the main paper. Since $\epsilon$ was used to denote the fractional difference between $k$ and $m_{a} / 2$, we have $\epsilon_{1}=$ $\epsilon /(4 \gamma)$ [see Eq. (A6)] so $\nu \eta= \pm \mu t$. Thus, the solution we obtained is (recall $\xi=t$ )

$$
\begin{aligned}
\Psi(t)= & C_{1}\left(\cos \left(\frac{m_{a}}{2} t\right)-\beta^{(s)} \sin \left(\frac{m_{a}}{2} t\right)\right) e^{\mu t} \\
& +C_{2}\left(-\beta^{(s)} \cos \left(\frac{m_{a}}{2} t\right)+\sin \left(\frac{m_{a}}{2} t\right)\right) e^{-\mu t}
\end{aligned}
$$

where

$$
\beta^{(s)} \equiv \frac{\lambda^{(s)}-\sqrt{1-16 \epsilon_{1}^{2}}}{4 \epsilon_{1}}
$$

and $C_{1}$ and $C_{2}$ are arbitrary constants.

Then we extract the forward and backward waves from Eq. (A16) by expressing cosine and sine functions in terms of exponentials, as

$$
\begin{aligned}
\Psi(t)= & {\left[D_{1}\left(1+i \beta^{(s)}\right) e^{\mu t}+D_{2}\left(1-i \beta^{(s)}\right) e^{-\mu t}\right] e^{i \frac{m_{a}}{2} t} } \\
& +\left[D_{1}\left(1-i \beta^{(s)}\right) e^{\mu t}-D_{2}\left(1+i \beta^{(s)}\right) e^{-\mu t}\right] e^{-i \frac{m_{a}}{2} t}
\end{aligned}
$$

where the arbitrary constants are redefined. Recalling that we started from Eq. (A1) about the spatial Fourier mode $\sim e^{i k x}$, the $e^{i \frac{m a}{2} t}$ part denotes the backward wave and the $e^{-i \frac{m_{a} t}{2} t}$ part is for the forward wave. We now put the initial condition of vanishing backward wave at $t=0$. We first 
write the $D$ coefficients as $D_{1}=D\left(1-i \beta^{(s)}\right)$ and $D_{2}=-D\left(1+i \beta^{(s)}\right)$, and normalize by the initial amplitude of the forward wave $h_{0}=2 D\left(1-\left(\beta^{(s)}\right)^{2}\right)$. This gives

$$
\begin{aligned}
\Psi(t)= & h_{0} \frac{1+\left(\beta^{(s)}\right)^{2}}{1-\left(\beta^{(s)}\right)^{2}} \sinh (\mu t) e^{i \frac{m_{a}}{2} t} \\
& +h_{0}\left[\cosh (\mu t)-i \frac{2 \beta^{(s)}}{1-\left(\beta^{(s)}\right)^{2}} \sinh (\mu t)\right] e^{-i \frac{m_{a}}{2} t} .
\end{aligned}
$$

To further simplify the coefficients, we recall Eq. (A17). Since $\lambda^{(s)}= \pm 1$, we have

$$
\begin{aligned}
\Psi(t)= & h_{0} \lambda^{(s)} \frac{\gamma}{\sqrt{\gamma^{2}-\epsilon^{2}}} \sinh (\mu t) e^{i \frac{m_{a}}{2} t} \\
& +h_{0}\left[\cosh (\mu t)-i \frac{\epsilon}{\sqrt{\gamma^{2}-\epsilon^{2}}} \sinh (\mu t)\right] e^{-i \frac{m_{a}}{2} t}
\end{aligned}
$$

This is the same solution as in Eqs. (13a) and (13b), considering the phase factors in the ansatz, Eq. (5). Since we assumed the initial phase of the axion field to be zero, it does not appear here. Also, here the initial amplitude of the forward wave $h_{0}$ is assumed to be real. Thus, we have obtained the identical solution via solving the Mathieu equation.

\section{APPENDIX B: SKETCH FOR THE AXION SEARCH IN THE FREQUENCY-TIME PLANE}

In the main part of this paper, we have used only pink elephant features in the frequency domain to quickly estimate the search prospect. To this end, we have imposed relatively strong detection criteria so that the ignorance of other realistic effects may not invalidate our results.

In this Appendix, we aim to provide a more complete discussion of the signal and its search in the frequency-time domain. We first show time domain features of the signal using spectrograms - the signal amplitude in the frequency-time dimension-obtained by simulating LIGO analysis. This shows what a signal would be like in the real data. Based on the spectrograms and calculations in the main text, we briefly suggest how LIGO search might be able to utilize full features in the frequency and time domains. Along with this, we also point out challenges (that we have simply assumed to be well resolved) that real LIGO analysis may have to deal with, and after all, we support our results in the main text.

We collect all five features that can/must be used to search for the axion signal:

(i) resonance peak frequency $f_{0}=m_{a} / 4 \pi$,

(ii) resonance frequency width $\simeq f_{0} \Delta v$, (iii) resonance amplification determined by $(N \delta(f))_{\text {halo }}$ as in Eq. (26),

(iv) time duration $\sim 1 / m_{a} \Delta v$,

(v) time delay of the peak $\simeq R \gamma^{2} / 12 \Delta v^{2}$, with $R \simeq$ $100 \mathrm{kpc}$ and $\gamma$ determined by Eq. (9).

These features are shown well in the spectrograms in Fig. 4, where the GW amplitude is color-coded in the frequencytime dimensions. They are obtained by simulating LIGO analysis as detailed below, so the actual signals may look in this way in the real data (after noise is removed). One can see, in addition to the standard chirping signals, an almost mono-frequency signal-narrow resonance in the frequency domain - with long time duration and time delay with respect to the chirping. The same signal will be observed for every GW event. Obviously, signals are strong enough and highly characteristic so that a proper analysis should be able to detect them by correlating all GW events.

Most important axion parameters are $m_{a}$ and $\ell$. The resonance frequency is exactly fixed to be $f_{0}=m_{a} / 4 \pi$, while the frequency width and time duration are determined together with $\Delta v \simeq 10^{-3}$. The remaining two-amplification and time delay - depend additionally on the actual path of the GW through the MW axion halo and the axion density along
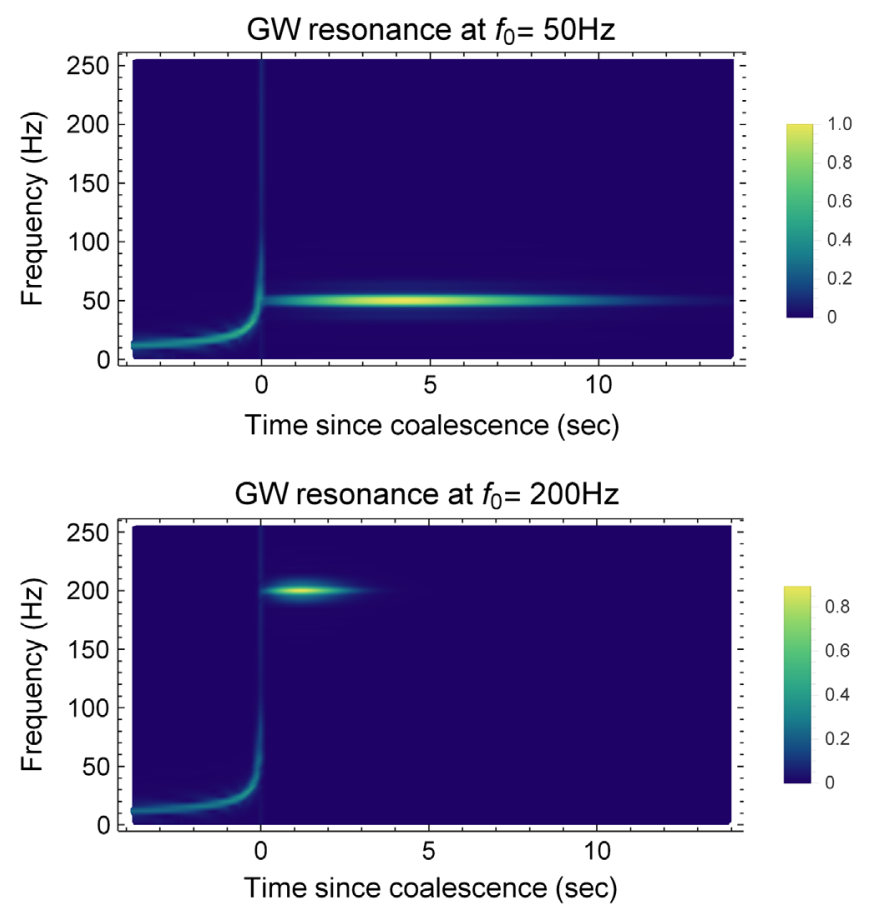

FIG. 4. Example spectrograms (normalized amplitudes) of axion signals in association with chirping GWs, for the resonance frequency $50 \mathrm{~Hz}$ (upper) and $200 \mathrm{~Hz}$ (lower). In the frequency domain, the signals are strong narrow peaks, while in the time domain, they are time delayed compared to the chirping part and have finite time duration. The spectrograms are obtained by simulating LIGO analysis with sampling frequency $2048 \mathrm{~Hz}$, $0.25 \mathrm{sec}$ time bin, $50 \%$ overlap between them, and the Tukey window with $\alpha=0.5$. Other details are as in Fig. 1; in particular, the coupling $\ell$ is chosen to just satisfy detection criteria. 
the path. However, the variations would not be significant given that axions are relatively uniformly distributed and the solar system is well inside the MW. A dedicated modeling of the MW axion halo can be used to obtain the possible range of them.

With these, we briefly suggest how LIGO search might be able to utilize all these features:

(1) The search starts from the detection of the standard chirping GWs as usual. For each GW event, we find signal candidates as follow.

(2) Select the target $m_{a}$ and $\ell$-these determine most features of the signal as discussed. Search for the signal following the steps below, and repeat the steps with other $m_{a}$ and $\ell$.

(3) Obtain the time series of data, long enough to include the expected duration of the axion signal $1 / m_{a} \Delta v(\sim \mathcal{O}(1-100) \mathrm{sec}$ at LIGO) and the expected time delay after the chirping signal (Fig. 3). Since those properties may vary slightly among GW events and signals are anyway weak at the beginning and the end in the time domain (see spectrograms), the exact values may not be so important as long as all signals can be measured to have similar duration and time delay.

(4) Obtain the power spectrum of the selected time series by the Fourier transform. If the axion signal is present, there should be a "significant" SNR gain peaked at the resonance frequency $f_{0}=m_{a} / 4 \pi$, with the amount corresponding to the expected amplification. The peak width $\sim f_{0} \Delta v$ may or may not be resolved well because the width is similar to the frequency resolution of the (discrete) Fourier transform of the selected time series.
(5) A true signal, if any, must be present in all GW events with almost the same properties; otherwise, the target $m_{a}$ and $\ell$ can be constrained. The correlation of all five features among all GW events must improve the likelihood of the signal.

The main assumptions that we have made in this paper were the selection of long enough time series, successful correlation among all GWs, and reduction of accidental noise peaks. Since we ignore these processes, we required a strong enough peak amplification [Eq. (28)] to be conservative in our estimation. How well these processes can be done in real LIGO analyses is beyond the scope of the paper; a dedicated study is encouraged. But given the strong characteristics of the signal, we believe that a careful analysis can work well. One example reducible noise is a sharp frequency noise from mechanical and instrumental reasons. Most of them would be random in its amplitude and timing by its nature, so that the correlation among number of GW events may be able to remove them. Even though some constant stationary noises (like the one coming from electricity) may still remain, they are small in the majority of the frequency band.

Lastly, we detail technical aspects of the spectrograms in Fig. 4. This is relevant to step 3 and 4. We dissect theoretical GW waveforms in the time domain with the sampling frequency $2048 \mathrm{~Hz}$, bin them with the time bin size $0.25 \mathrm{sec}$ and $50 \%$ overlap (i.e., $0.125 \mathrm{sec}$ overlap) between neighboring bins, and weight sum each bin by the Tukey window function with $\alpha=0.5$. The discrete Fourier transform of the resulting time series of data yields the spectrograms.
[1] A. Arvanitaki, S. Dimopoulos, S. Dubovsky, N. Kaloper, and J. March-Russell, String axiverse, Phys. Rev. D 81, 123530 (2010).

[2] P. W. Graham and A. Scherlis, Stochastic axion scenario, Phys. Rev. D 98, 035017 (2018).

[3] F. Takahashi, W. Yin, and A. H. Guth, QCD axion window and low-scale inflation, Phys. Rev. D 98, 015042 (2018).

[4] P. W. Graham and S. Rajendran, Axion dark matter detection with cold molecules, Phys. Rev. D 84, 055013 (2011).

[5] D. J. E. Marsh, Axion cosmology, Phys. Rep. 643, 1 (2016).

[6] K. Choi, J. c. Hwang, and K. W. Hwang, String theoretic axion coupling and the evolution of cosmic structures, Phys. Rev. D 61, 084026 (2000).

[7] J.E. Kim, Gravity wave and model-independent axion, J. Korean Phys. Soc. 71, 127 (2017).

[8] Y. Ali-Haimoud and Y. Chen, Slowly-rotating stars and black holes in dynamical Chern-Simons gravity, Phys. Rev. D 84, 124033 (2011).
[9] G. F. Giudice, M. McCullough, and A. Urbano, Hunting for dark particles with gravitational waves, J. Cosmol. Astropart. Phys. 10 (2016) 001.

[10] S. Jung and C. S. Shin, Gravitational-Wave Fringes at LIGO: Detecting Compact Dark Matter by Gravitational Lensing, Phys. Rev. Lett. 122, 041103 (2019).

[11] K. H. Lai, O. A. Hannuksela, A. Herrera-Martin, J. M. Diego, T. Broadhurst, and T. G. F. Li, Discovering intermediate-mass black hole lenses through gravitational wave lensing, Phys. Rev. D 98, 083005 (2018).

[12] P. Christian, S. Vitale, and A. Loeb, Detecting stellar lensing of gravitational waves with ground-based observatories, Phys. Rev. D 98, 103022 (2018).

[13] L. Dai, S. S. Li, B. Zackay, S. Mao, and Y. Lu, Detecting lensing-induced diffraction in astrophysical gravitational waves, Phys. Rev. D 98, 104029 (2018).

[14] S. Jung and T. Kim, Probing cosmic strings with gravitational-wave lensing fringe, J. Cosmol. Astropart. Phys. 07 (2020) 068. 
[15] H. G. Choi and S. Jung, New probe of dark matter-induced fifth force with neutron star inspirals, Phys. Rev. D 99, 015013 (2019).

[16] D. Yoshida and J. Soda, Electromagnetic waves propagating in the string axiverse, Prog. Theor. Exp. Phys. 2018, $041 \mathrm{E} 01$ (2018).

[17] A. Arza, Photon enhancement in a homogeneous axion dark matter background, Eur. Phys. J. C 79, 250 (2019).

[18] A. Caputo, C. P. Garay, and S. J. Witte, Looking for axion dark matter in dwarf spheroidals, Phys. Rev. D 98, 083024 (2018); Erratum, Phys. Rev. D 99, 089901 (2019).

[19] A. Caputo, M. Regis, M. Taoso, and S. J. Witte, Detecting the stimulated decay of axions at radio frequencies, J. Cosmol. Astropart. Phys. 03 (2019) 027.

[20] J. G. Rosa and T. W. Kephart, Stimulated Axion Decay in Superradiant Clouds Around Primordial Black Holes, Phys. Rev. Lett. 120, 231102 (2018).

[21] A. Arza and P. Sikivie, Production and Detection of an Axion Dark Matter Echo, Phys. Rev. Lett. 123, 131804 (2019).

[22] M. P. Hertzberg and E. D. Schiappacasse, Dark matter axion clump resonance of photons, J. Cosmol. Astropart. Phys. 11 (2018) 004.

[23] Z. Wang, L. Shao, and L.-X. Li, Resonant instability of axionic dark matter clumps, J. Cosmol. Astropart. Phys. 07 (2020) 038.

[24] P. Carenza, A. Mirizzi, and G. Sigl, Dynamical evolution of axion condensates under stimulated decays into photons, Phys. Rev. D 101, 103016 (2020).

[25] I. I. Tkachev, Fast radio bursts and axion miniclusters, Pis'ma Zh. Eksp. Teor. Fiz. 101, 3 (2015) [JETP Lett. 101, 1 (2015)].

[26] D. Yoshida and J. Soda, Exploring the string axiverse and parity violation in gravity with gravitational waves, Int. J. Mod. Phys. D 27, 1850096 (2018).

[27] S. Sun and Y. L. Zhang, Gravitational waves and possible fast radio bursts from axion clumps, arXiv:2003.10527.

[28] C. S. Chu, J. Soda, and D. Yoshida, Gravitational waves in axion dark matter, Universe 6, 89 (2020).

[29] N. Kitajima, J. Soda, and Y. Urakawa, Gravitational wave forest from string axiverse, J. Cosmol. Astropart. Phys. 10 (2018) 008.

[30] S. Alexander and J. Martin, Birefringent gravitational waves and the consistency check of inflation, Phys. Rev. D 71, 063526 (2005).

[31] S. Alexander and N. Yunes, Chern-Simons modified general relativity, Phys. Rep. 480, 1 (2009).

[32] M. Okounkova, L. C. Stein, M. A. Scheel, and D. A. Hemberger, Numerical binary black hole mergers in dynamical Chern-Simons gravity: Scalar field, Phys. Rev. D 96, 044020 (2017).

[33] S. Tulin and H. B. Yu, Dark matter self-interactions and small scale structure, Phys. Rep. 730, 1 (2018).

[34] E. W. Kolb and I. I. Tkachev, Axion Miniclusters and Bose Stars, Phys. Rev. Lett. 71, 3051 (1993).

[35] J. Preskill, M. B. Wise, and F. Wilczek, Cosmology of the invisible axion, Phys. Lett. 120B, 127 (1983).

[36] L. F. Abbott and P. Sikivie, A cosmological bound on the invisible axion, Phys. Lett. 120B, 133 (1983).

[37] M. Dine and W. Fischler, The not so harmless axion, Phys. Lett. 120B, 137 (1983).

[38] F. Nesti and P. Salucci, The dark matter halo of the Milky Way, AD 2013, J. Cosmol. Astropart. Phys. 07 (2013) 016.

[39] C. Cutler and E.E. Flanagan, Gravitational waves from merging compact binaries: How accurately can one extract the binary's parameters from the inspiral wave form?, Phys. Rev. D 49, 2658 (1994).

[40] I. G. Irastorza and J. Redondo, New experimental approaches in the search for axion-like particles, Prog. Part. Nucl. Phys. 102, 89 (2018).

[41] D. J. Griffiths, Introduction to Quantum Mechanics (Pearson Education, New Jersey, 2005).

[42] P. C. Peters, Index of refraction for scalar, electromagnetic, and gravitational waves in weak gravitational fields, Phys. Rev. D 9, 2207 (1974).

[43] M. M. Ivanov, Y. Y. Kovalev, M. L. Lister, A. G. Panin, A. B. Pushkarev, T. Savolainen, and S. V. Troitsky, Constraining the photon coupling of ultra-light dark-matter axion-like particles by polarization variations of parsecscale jets in active galaxies, J. Cosmol. Astropart. Phys. 02 (2019) 059.

[44] D. Harari and P. Sikivie, Effects of a Nambu-Goldstone boson on the polarization of radio galaxies and the cosmic microwave background, Phys. Lett. B 289, 67 (1992).

[45] M. A. Fedderke, P. W. Graham, and S. Rajendran, Axion dark matter detection with CMB polarization, Phys. Rev. D 100, 015040 (2019).

[46] A. Lue, L. M. Wang, and M. Kamionkowski, Cosmological Signature of New Parity Violating Interactions, Phys. Rev. Lett. 83, 1506 (1999).

[47] G. Sigl and P. Trivedi, Axion-like dark matter constraints from CMB birefringence, arXiv:1811.07873.

[48] I. Kovacic, R. Rand, and S. M. Sah, Mathieu's equation and its generalizations: Overview of stability charts and their features, Appl. Mech. Rev. 70, 020802 (2018).

[49] R. H. Rand and D. Armbruster, Perturbation Methods, Bifurcation Theory and Computer Algebra, Applied Mathematical Sciences, Vol. 65 (Springer-Verlag, New York, 1987). 